\title{
ESPR uroradiology task force and ESUR paediatric working group: imaging recommendations in paediatric uroradiology, part IV
}

\section{Minutes of the ESPR uroradiology task force mini-symposium on imaging in childhood renal hypertension and imaging of renal trauma in children}

\author{
Michael Riccabona - M. L. Lobo - F. Papadopoulou • \\ F. E. Avni - J. G. Blickman - J. N. Dacher • B. Damasio • \\ K. Darge • L. S. Ording-Müller • P. H. Vivier • U. Willi
}

Received: 24 January 2011 / Accepted: 4 April 2011

(C) Springer-Verlag 2011

\begin{abstract}
Two new recommendations of the European Society of Radiology task force and the European Society of Uroradiology workgroup on paediatric uroradiology are presented. One deals with diagnostic imaging in children after trauma to the urinary tract - renal trauma, in particular. The other concerns the evaluation of suspected renal hypertension. Available data in the paediatric literature are either unsatisfactory or controversial for both of these clinical settings. Therefore, the following consensus-based proposals aim at outlining effective imaging algorithms to reduce invasive
\end{abstract}

M. Riccabona $(\bowtie)$

Department of Radiology, Division of Pediatric Radiology,

University Hospital Graz,

Auenbruggerplatz, 8036 Graz, Austria

e-mail: michael.riccabona@klinikum-graz.at

\section{L. Lobo}

Department of Radiology,

Hospital de Santa Maria, University Hospital,

Lisbon, Portugal

F. Papadopoulou

Department of Radiology, Ioannina University Hospital,

Ioannina, Greece

F. E. Avni

Departments of Medical Imaging,

University Clinics of Brussels-Erasme Hospital,

Brussels, Belgium

J. G. Blickman

Department of Radiology, University of Rochester,

School of Medicine,

Rochester, NY, USA imaging procedures while optimising diagnostic accuracy. The objective of following a more uniform imaging approach is to facilitate future meta-analysis as well as multicentre and other more evidence-based studies. The practise in paediatric radiology is typically based on local availability and on the limitations of professional expertise and equipment, balanced against the perceived needs of the individual child. Although this is unlikely to change in the near future, it does not release the physicians in charge of diagnostic imaging from their responsibility in choosing and providing state-of-the-art

J. N. Dacher $\cdot$ P. H. Vivier

Department of Radiology, CHU de Rouen,

Rouen, France

B. Damasio

Department of Radiology, Gaslini Institute Genoa,

Genoa, Italy

K. Darge

Department of Radiology, Children's Hospital of Philadelphia, Philadelphia, PA, USA

L. S. Ording-Müller

Department of Radiology, University Hospital North Norway, Tromsø, Norway

U. Willi

Department of Radiology, Johns Hopkins University, Baltimore, MD, USA 
imaging and management protocols that are adapted specifically for use in children.

Keywords Hypertension · Renal artery stenosis $\cdot$ Renal trauma $\cdot$ Urinary tract $\cdot$ Children

\section{Introduction and rationale}

Consistent effort made by the European Society of Paediatric Radiology (ESPR) uroradiology task force and the European Society of Uroradiology (ESUR) paediatric uroradiology workgroup over recent years has resulted in the publication of several imaging and procedural recommendations.

The aspects of paediatric uroradiology covered thus far include imaging of the following conditions: prenatally diagnosed moderate and high-grade hydronephrosis (HN), childhood urolithiasis and haematuria, paediatric obstructive uropathy, and urinary tract infection.

Additionally, procedural recommendations for the following commonly indicated paediatric uroradiology procedures have been issued: paediatric urosonography, contrast-enhanced voiding urosonography, voiding cystourethrography, intravenous urography, childhood uro-computed tomography (CT) and magnetic resonance (MR)-urography. A separate statement on childhood aspects of nephrogenic systemic fibrosis has been issued and a standardised adapted grading scheme for hydronephrosis has been presented. These recommendations have gained increasing acceptance throughout Europe and are being discussed in the wider paediatric radiology community.

To complete the existing recommendations, the joint working group has now addressed two additional controversial, yet important, topics: imaging recommendations in childhood hypertension and in paediatric renal trauma. The objective was again to standardise paediatric uro-imaging procedures and to minimise invasiveness and radiation without sacrificing the diagnostic accuracy for causative pathology in the individual patient.

Similar to the previous recommendations, there is a paucity of evidence, and the new proposals are again consensus-based after a thorough review of the literature and existing guidelines and recommendations. The proposals have been thoroughly discussed in the respective societies as well as with nonmember experts in the field and with colleagues from other
Fig. 1 Imaging algorithm in (a) severe and (b) mild-to-moderate paediatric urinary tract trauma. If vascular complications are suspected, angiography/embolisation should be considered. Potentially, MRI could be used for late presentation and for follow-up. It is important to avoid unnecessary repeated CT. 1 Use voiding cystouretrography for (suspected) injury to the urinary bladder. Late phase contrastenhanced CT/CT-cystography should be used only if CT is already done on other indications. 2 See also previous procedural recommendations. 3 Amplitude-coded colour Doppler sonography (CDS; potentially with intravenous contrast administration) is the first choice modality for follow-up. DMSA Dimercaptosiccinidacid static renal scintigraphy, $C T$ computed tomography, MRI magnetic resonance imaging, US ultrasound a Imaging the child following major urinary tract trauma

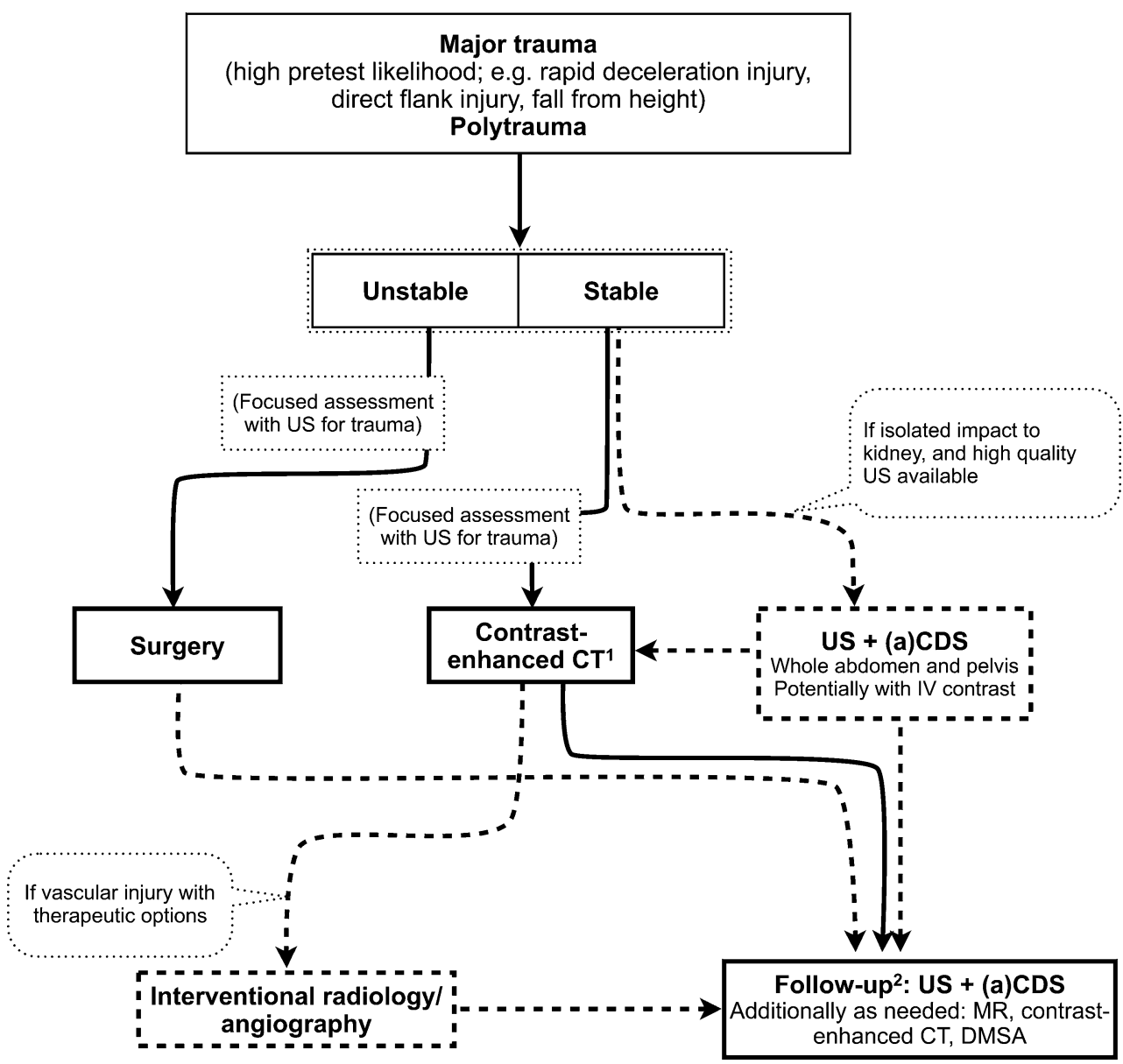


partner disciplines, e.g., paediatricians, paediatric nephrologists, surgeons and urologists.

Based on the above, the following two recommendations have been drafted and presented as well as discussed during the mini-symposium "Paediatric uroradiology imaging recommendations, part IV" at the annual ESPR meeting in June 2010 in Bordeaux, France. This ensured the more general opinion was also heard. Subsequent feedback obtained at the guideline session of the annual ESUR meeting in September 2010 in Bruges, Belgium, was included in the finalising of the new recommendations.

It is hoped that these proposed recommendations will help streamline optimal imaging procedures in paediatric uroradiology throughout Europe, in spite of remaining concerns and unresolved issues. This is a prerequisite to facilitate the meta-analysis of results from future multicentre studies, and a step towards obtaining more evidence-based data.

\section{Imaging children with renal trauma}

Children have a higher risk of renal lesions than adults following blunt abdominal trauma. This is due to age-related increased organ mobility, relatively larger size and less anatomical protection of the kidneys.

Gross or microscopic haematuria is a common presenting symptom, but the degree of haematuria does not necessarily correlate with the presence or severity of possible renal injury; therefore, every child with haematuria after abdominal trauma should undergo renal imaging.

$\mathrm{CT}$ is considered the imaging modality of choice in severe abdominal trauma; its use at first-line evaluation is unequivocally accepted in haemodynamically stable children, especially in suspected spinal or pelvic girdle involvement, and suspected rupture of the urinary bladder (Fig. 1a). CT is also the first choice if US is not possible, e.g., due to skin lesions, bandages or in obese or restless patients. CT depicts most lesions that may escape

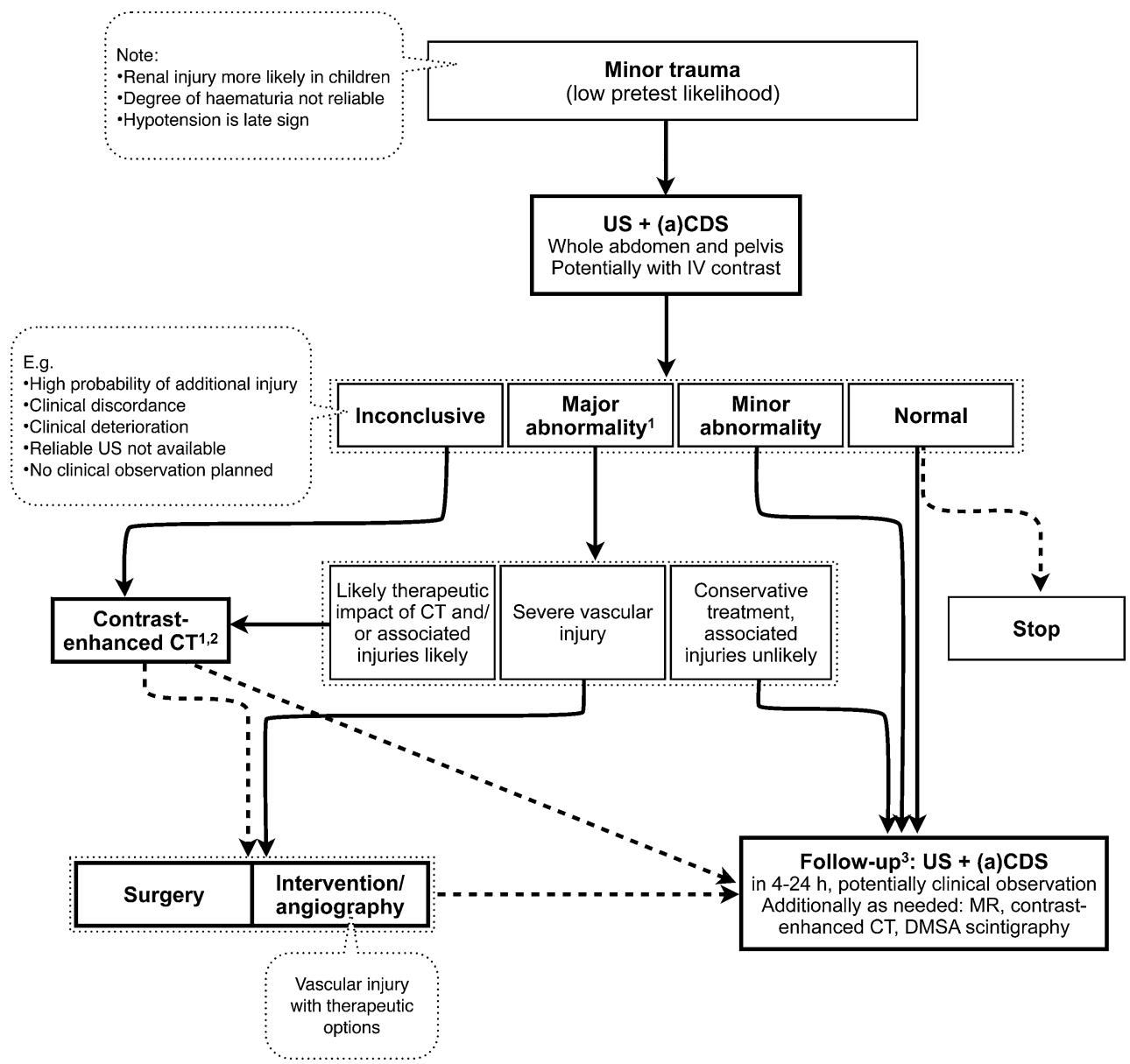

Fig. 1 (continued) 
US detection. It is also the safest, most reliable and most widely available method to exclude significant urinary tract injuries.

Of special concern in children, however, is the inevitable and significant radiation exposure. Other disadvantages include the need for intravenous iodinated contrast medium administration and the potential risk related to transporting an injured patient.

On the other hand, a comprehensive US examination including colour and spectral Doppler analysis is often sufficient to reliably exclude major renal injury when performed under favourable conditions and by an experienced examiner using adequate equipment. Minor renal lesions, e.g., a subtle contusion that would not have therapeutic consequences, may be missed by US. Therefore, US may be the initial choice of paediatric renal imaging in minor or moderate trauma. Complementary CT should be considered in cases in which US is inconclusive or discordant with more concerning clinical findings, if there is clinical deterioration, or if the findings by US indicate further imaging in view of directing therapy and management (Fig. 1b). On the other hand, US should certainly be considered as the method of choice in the follow-up of children with traumatic renal lesions, even if initially evaluated by $\mathrm{CT}$. If additional cross-sectional imaging is needed during follow-up in the nonemergency setting, MRI should be used whenever available, especially if US does not offer conclusive results.

\section{Imaging in suspected childhood renal hypertension}

Renovascular hypertension is rare in children. However, unlike in adults, renovascular is more common than idiopathic hypertension. There is an ongoing debate as to how to image children with clinically suspected renovascular hypertension. The use of noninvasive CT angiography (CTA) is particularly controversial. MR angiography (MRA) is feasible in older children and is relatively sensitive for significant stenoses of the main renal artery, but less sensitive in smaller children and for the more peripheral interlobar and arcuate arteries due to the lower temporal and spatial resolution.
Fig. 2 Imaging algorithm in children with suspected renovascular hypertension. 1 High clinical suspicion: history of renal trauma or radiation, umbilical artery catheterisation, renovascular thrombosis, bruit over renal arteries, high renin levels, presence of disease associated with renovascular abnormality (e.g. neurofibromatosis, Williams syndrome,

tuberous sclerosis).

2 See previous

recommendations.

3 Potentially and increasingly contrast-enhanced MR angiography (MRA) can be used for assessment of larger vessels and infarcted areas. Non-enhanced MRA techniques are emerging. 4 Captopril scintigraphy may potentially be used prior to percutaneous transluminal angioplasty (PTA) for functional assessment, particularly in doubtful cases, or in neonates until old enough for PTA; note local variations in practice. $B P$ blood pressure, $C D S$ colour Doppler sonography, CTA computed tomography angiography, $D D S$ spectral duplex Doppler, $D S A$ digital subtraction angiography, $P S V$ peak systolic velocity, $R A R$ renal-aortic ratio, $\delta-R I$ resistive index difference

\section{Imaging the child with suspected renovascular hypertension}

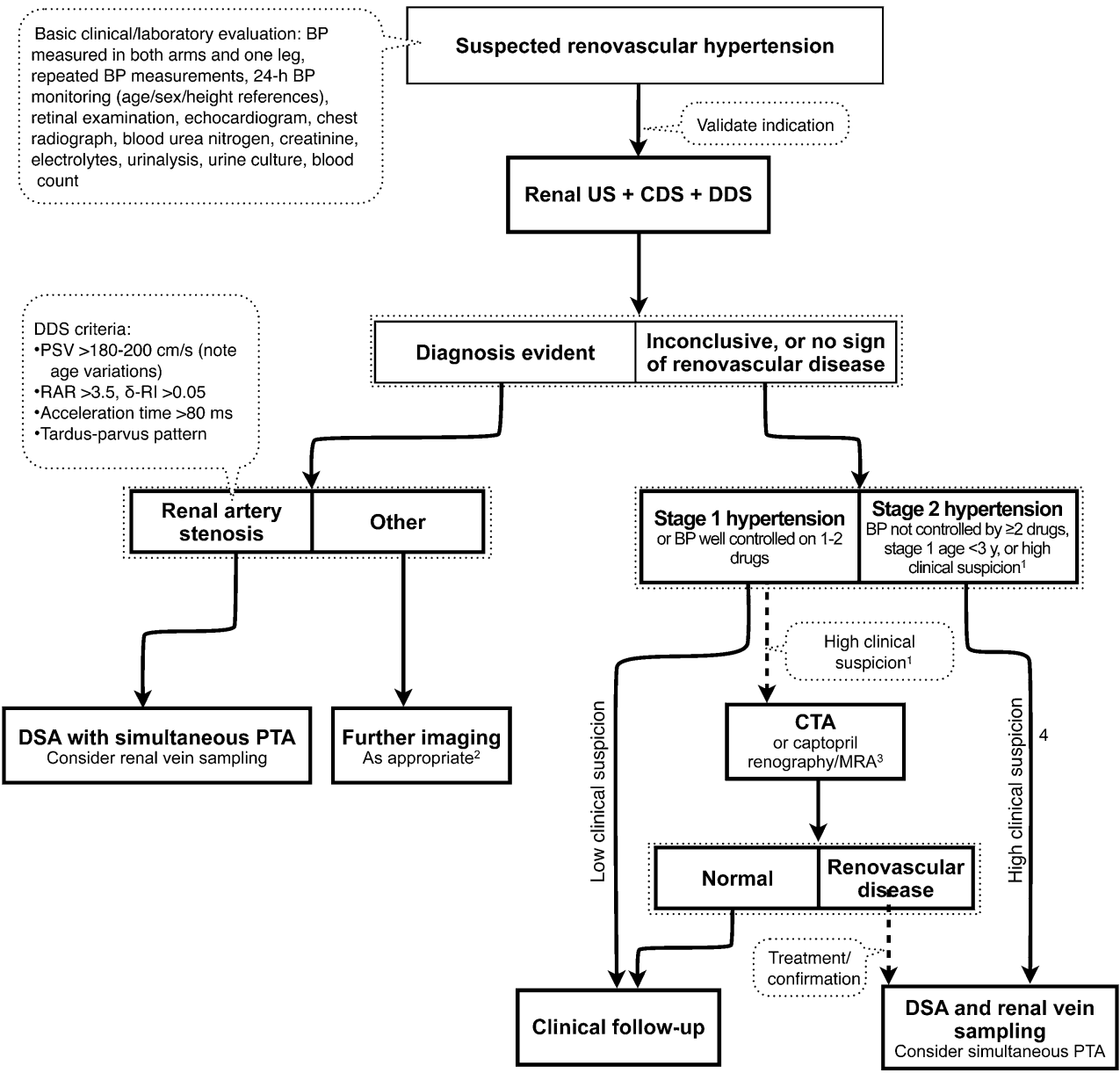


Various Doppler findings and flow parameters have not been widely or adequately evaluated for diagnostic performance in childhood renal arterial stenosis (RAS). It is not clear whether the accepted criteria in adults, i.e. a peak systolic velocity $>180-200 \mathrm{~cm} / \mathrm{s}$, acceleration time $>80 \mathrm{~ms}$ (both parameters show age dependent variations in early childhood), difference of resistive index $>0.05$, and renal artery to aortic flow velocity ratio $>3-3.5$, are applicable to children. Only the typical distal pulsus tardus and parvus waveform and the direct visualisation of the stenosis on CDS (turbulent flow at increased velocity causing aliasing) are reliable predictors for childhood RAS.

Given the above information and the variable availability of state-of the-art equipment, it is difficult to establish an effective, economic, reliable, evidence-based imaging algorithm, particularly if one wants to favour and promote the use of noninvasive and nonionising techniques.

The current recommendation is that children with a high probability of renovascular disease, a positive CDS study and severe disease should, following comprehensive US evaluation, be referred for catheter angiography with renal vein sampling, potentially with simultaneous endovascular treatment. Further noninvasive imaging by CTA or MRA has no significant benefit in these children; it only creates additional costs and may introduce delay.

This strategy, however, is under discussion in equivocal cases. It will likely have to undergo revision with respect to the most recent multidetector-CT technology allowing for digital subtraction CTA and thus evaluation of smaller intrarenal vessels.

Continued (multicentre) research is necessary in various aspects of renal vascular evaluation. Research topics should include the predictive value of various Doppler indices in childhood for different age groups, the potential of modern CTA techniques in children and the use of nonenhanced MRA techniques in children. The efficacy of these needs to be re-evaluated: Are they reliable and how do they impact on therapy and prognosis?

Selection of those patients who present with mild to moderate or unclear disease, or who have a low probability of significant RAS based on US, CDS and clinical information prior to the more invasive test by CTA (or, possibly, captopril scintigraphy) may be useful (Fig. 2).

Follow-up of patients who have undergone treatment for RAS has not been addressed with this algorithm.

\section{Conclusion}

The joint ESPR task force and ESUR workgroup on paediatric uroradiology propose two new imaging algo- rithms with the aim of improving and standardising imaging in the child following urinary tract trauma and in the child with suspected renovascular hypertension.

In severe urinary tract trauma, CT is the primary modality, whereas in less severe or minimal trauma, a comprehensive US evaluation including CDS may often suffice for initial evaluation and for virtually all follow-up. Controversy continues regarding the most appropriate imaging modality and pathway for imaging children with suspicion of renovascular hypertension. Angiography with vascular intervention offers the highest diagnostic accuracy with the option of simultaneous treatment. CDS, captopril scintigraphy, MRA, CTA or a combination of these should be considered prior to angiography, especially in questionable cases or as an initial investigation in children with lower probability for significant renovascular disease.

Acknowledgement We thank Dr D. Roebuck, Great Ormond Street Hospital for Children, London, United Kingdom, for his valuable contribution and support in preparing the hypertension algorithm.

Disclosures M.R. participates in research collaboration with, and is a consultant for, GE (US) and Toshiba (CT).

\section{References}

1. Brenner DJ, Hall EJ (2007) Computed tomography: an increasing source of radiation exposure. N Engl J Med 357:2277-2284

2. Brun Ph, Kchouk H, Mouchet B et al (1997) Value of Doppler ultrasound in the diagnosis of renal artery stenosis in children. Pediatr Nephrol 11:27-30

3. Dacher JN, Cellier C (2008) Urinary tract trauma. In: Fotter R (ed) Paediatric uroradiology, 2nd revised edn. Springer, New York, pp 461-472

4. Deeg KH, Wörle K, Wolf A (2003) Doppler sonographic estimation of normal values for flow velocity and resistance indices in renal arteries of healthy infants (in German). Ultraschall Med 24:312-322

5. Eeg KR, Khoury AE, Halachmi S et al (2009) Single center experience with application of the ALARA concept to serial imaging studies after blunt renal trauma in children: is ultrasound enough? J Urol 181:1834-1840

6. Hom $J$ (2010) The risk of intra-abdominal injuries in paediatric patients with stable blunt abdominal trauma and negative abdominal computed tomography. Acad Emerg Med 17:469-475

7. Kawashima A, Sandler CM, Corl FM et al (2001) Imaging of renal trauma: a comprehensive review. Radiographics 21:557-574

8. Nghia JV, Hammelman BD, Racadio JM et al (2006) Anatomic distribution of renal artery stenosis in children: implications for imaging. Pediatr Radiol 36:1032-1036

9. Patriquin H (1991) Doppler examination of the kidney in infants and children. Urol Radiol 12:220-227

10. Pietrera P, Badachi Y, Liard A et al (2001) Ultrasound for initial evaluation of post-traumatic renal lesions in children (in French). J Radiol 82:833-838

11. Reusz GS, Kis E, Cseprekal O et al (2010) Captopril-enhanced renal scintigraphy in the diagnosis of pediatric hypertension. Pediatr Nephrol 25:185-189

12. Riccabona M, Avni F, Blickman J et al (2008) Imaging recommendations in paediatric uroradiology: minutes of the ESPR 
workgroup session on urinary tract infection, fetal hydronephrosis, urinary tract ultrasonography and voiding cystourethrography. ESPR-Meeting, Barcelona, Spain, June 2007. Pediatr Radiol 38:138-145

13. Riccabona M, Avni FE, Dacher JN et al (2010) ESPR uroradiology task force and ESUR paediatric working group: imaging and procedural recommendations in paediatric uroradiology, part III. Minutes of the ESPR uroradiology task force minisymposium on intravenous urography, uro-CT and MR-urography in childhood. Pediatr Radiol 40:1315-1320

14. Riccabona M, Avni FE, Blickman JG et al (2009) Imaging recommendations in paediatric uroradiology, part II: urolithiasis and haematuria in children, paediatric obstructive uropathy, and postnatal work-up of foetally diagnosed high grade hydroneph- rosis. Minutes of a mini-symposium at ESPR annual meeting. Pediatr Radiol 39:891-898

15. Roebuck D (2008) Childhood hypertension: what does the radiologist contribute? Pediatr Radiol 38(Suppl 3):S501-S507

16. Tullus K, Brennan E, Hamilton G et al (2008) Renovascular hypertension in children. Lancet 371:1453-1463

17. Tullus K, Roebuck DJ, McLaren CA et al (2010) Imaging in the evaluation of renovascular disease. Pediatr Nephrol 25:1049-1056

18. Roth CG, Spottswood SE, Chan JC et al (2003) Evaluation of the hypertensive infant: a rational approach to diagnosis. Radiol Clin North Am 41:931-944

19. Wessel LM, Scholz S, Jester I et al (2000) Management of kidney injuries in children with blunt abdominal trauma. J Pediatr Surg 35:1326-1330 